\title{
Computergestützte Evaluation der Lehre
}

\author{
OVG Berlin, Beschluß vom 18. Juli 1991 (OVG PV Bln 9.91)
}

\section{Leitsätze der Redaktion}

1. Eine Leistungsüberwachung im Sinne des Personalvertretungsrechts liegt auch dann vor, wenn, wie im Falle des Projekts "Evaluation der Lehre” der Technischen Universität Berlin, die Leistung der Tutoren durch Studenten bewertet wird, mit dem Ziel, die Tutoren auf der Grundlage der gewonnenen Ergebnisse zur Überprüfung ihres Lehrverhaltens anzuregen. Auch die Tatsache, daß die Ergebnisse in individualisierter Form weder der Personalabteilung noch den Fachbereichen zugänglich gemacht werden, sondern nur den betroffenen Tutoren, vermag hieran nichts zu ändern.

2. Ein Mitbestimmungsrecht liegt, unabhängig davon, ob der Überwachungseffekt nach dem Willen des Dienststellenleiters ausgenutzt werden soll oder nicht, bereits dann vor, wenn die technische Einrichtung abstrakt in der Lage ist, die Überwachung von Dienstkräften zu ermöglichen (objektiv-finale Theorie).

\section{Gründe}

I.

Aufgrund eines Beschlusses des Akademischen Senats der Technischen Universität Berlin beabsichtigt der Beteiligte, im Rahmen des Projekts „Evaluation der Lehre” die Qualität der Lehrveranstaltungen in allen Fachbereichen der Universität durch die Studierenden bewerten zu lassen, um konkrete Studienreformmaßnahmen einleiten zu können. Zu diesem Zweck verteilen die Dozenten der einzelnen Lehrveranstaltungen an ihre Studenten Fragebogen, die von den Studenten ausgefüllt und danach wieder eingesammelt werden. Die Auswertung soll mit Hilfe der EDV-Anlage der Universität und eines dafür geeigneten Software-Programms erfolgen.

Nach der Darstellung des Beteiligten soll durch das Projekt herausgefunden werden, auf welche Weise durch Befragungen von Studierenden systematische und tiefenscharfe Informationen über die Qualität der Lehrveranstaltungen an der TU aus studentischer Sicht gewonnen werden können. Solche Informationen sollen dazu dienen, den Dozenten eine konkrete und differenzierte Rückmeldung darüber zu geben, wie die Lehrveranstaltung angekommen ist. Es soll versucht werden, Faktoren zu identifizieren, an denen eine Verbesserung der Lehrer ansetzen muß. Die Ergebnisse würden weder der Personalabteilung mitgeteilt werden, noch würden die Fachbereiche über einzelne Veranstaltungen informiert. Nur der jeweilige Dozent erhalte die Auswertung zu seiner persönlichen Verwendung.

Der Fragebogen beginnt mit der jeweiligen Lehrveranstaltungsnummer und enthält $u$. a. folgende Fragen:

- War der Dozent regelmäßig anwesend?

- Ist die Lehrveranstaltung manchmal ausgefallen?

- Der Dozent hat sich gut auf die Lehrveranstaltung vorbereitet.

- Ich halte den Dozenten für einen guten Lehrer.

- Der Dozent kümmert sich zu wenig um die Lehre.

- Wie beurteilen Sie die eingesetzten Skripte/Übungsblätter?

Als Antwort waren auf einer Skala Möglichkeiten von vollständiger Zustimmung bis zu vollständiger Ablehnung vorgesehen. Der Fragebogen ist für eine Auswertung durch elektrische Datenverarbeitung geeignet.

Nachdem der Beteiligte ein Mitbestimmungsrecht des Antragstellers bestritten hatte, hat dieser das personalvertretungsrechtliche Beschlußverfahren mit dem Ziel eingeleitet, sein Mitbestimmungsrecht nach $\$ 85$ Abs. 1 Nr. 13 PersVG Bin festzustellen. In dem entsprechenden Hauptverfahren VG FK (Bin) -C-5.91 ist eine Entscheidung bisher nicht ergangen.

Das Projekt "Evaluation der Lebre" an der TU Berlin

Nur der Dozent erbält die Auswertung zur persönlichen Verwendung.

Aus dem Fragebogen

Fragebögen für elektronische Auswertung geeignet

Mitbestimmungrecht nach PersVG Bln 


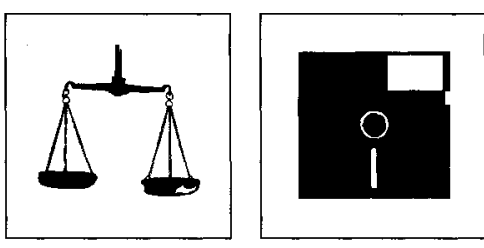

Die Entscheidung des VG

Beschwerdebegründung des Antragstellers

Antrag auf.Einleitung des Mitbestimmungsverfabrens

Vortrag der Antragsgegnerin

Zulässigkeit

Antragsbefugnis

Im vorliegenden Verfahren beantragt der Antragsteller,

dem Beteiligten im Wege dar einstweiligen Verfügung aufzugeben, das Mitbestimmungsverfahren im Zusammenhang mit dem Einsatz und der Auswertung des Fragebogens „Evaluation der Lehre" einzuleiten.

Dies hat das Verwaltungsgericht mit Beschluß vom 19. April 1991 abgelehnt und zur Begründung ausgeführt, zum einen sei der Erlaß der begehrten einstweiligen Verfügung nicht notwendig, da dem Antragsteller das behauptete Recht nicht verloren gehe, wenn er darauf verwiesen werde, es im Hauptverfahren durchzusetzen. Eine bis dahin eventuell bereits erfolgte Auswertung der Fragebögen könne dadurch rückgängig gemacht werden, daß die gewonnenen Daten wieder gelöscht würden. Zum anderen sei ein Obsiegen des Antragstellers im Hauptverfahren eher unwahrscheinlich. Das Mitbestimmungsrecht nach $\$ 85$ Abs. 1 Nr. 13 PersVG setze voraus, daß die technisierte Verhaltens- oder Leistungsüberwachung auf Daten beruhe, die hierzu überhaupt geeignet seien. Dafür ungeeignet seien Bewertungen durch Personen, die zu einer objektiven Beurteilung generell nicht in der Lage seien. Die Einschätzung von Lehrveranstaltungen durch Studenten dürfte zu dieser Kategorie ungeeigneter Daten gehören.

Zur Begründung seiner Beschwerde vertritt der Antragsteller die Auffassung, eine einstweilige Verfügung sei erforderlich, $\mathrm{da}$ anderenfalls von einem tatsächlich wirksamen Rechtsschutz nicht mehr ausgegangen werden könne. Im übrigen beziehe sich das Mitbestimmungsrecht nicht nur auf die Auswertung der erhobenen Daten, sondern bereits auf ihre Speicherung. Es handele sich auch entgegen dem angefochtenen Beschluß um überwachungsgeeignete Daten, da aus ihnen Schlüsse auf die Qualität von Lehrveranstaltungen gezogen werden sollten.

Der Antragsteller beantragt,

unter Aufhebung des Beschlusses des Verwaltungsgerichts Berlin vo'm 19. April 1991 dem Beteiligten im Wege der einstweiligan Verfügung aufzugeben, gegenüber dem Antragsteller das Mitbestimmungsverfahren bezüglich der EDV-gestützten Verarbeitung der Daten aus der Fragebogenumfrage zur Evaluation der Lehre an der Technischen Universität Berlin einzuleiten.

Der Antragsgegner beantragt,

die Beschwerde zurückzuweisen.

Er hält den Antrag auf Erlaß einer einstweiligen Verfügung mit dem Inhalt, ihm aufzugeben, eine Handlung zu unterlassen, die Vornahme einer Handlung zu dulden oder eine Handlung vorzunehmen für unzulässig. Im übrigen verweist er darauf, daß sowohl nach dem Arbeits- als auch nach dem Beamtenrecht Leistungsbewertungen nur durch den jeweiligen Vorgesetzten vorgenommen werden dürften. Weder Aussagen von Untergebenen noch von Dritten dürfe der Dienstvorgesetzte heranziehen, um eine Leistungsbewertung unkontrolliert durchzuführen. Die Vorgesetzten der hier betroffenen Tutoren seien die Professoren, nicht aber der Präsident der Universität, der für die Lehre nicht zuständig sei. Wegen der weiteren Einzelheiten wird auf den Inhalt der zwischen den Beteiligten gewechselten Schriftsätze Bezug genommen.

II.

Der Antrag ist zulässig. Der Charakter des personalvertretungsrechtlichen Beschlußverfahrens als eines objektiven Verfahrens steht nach der Rechtsprechung des Bundesverwaltungsgerichts zwar einem materiell-rechtlichen Unterkssungsanspmah entgegen, kina aber nicht den Erlaß einer einstweiligen Verfügung mit einem Ausspruch verfahrensrechtlichen Inhaltes in dem Sinne, daß er sich nur auf Verfahrenshandlungen bezieht (BVerwG 6 PB 12.89 - Beschluß vom 27. Juli 1990 = ZBR 1990, 354 = PersV 1990, 71; so schon OVG Berlin - OVG PV Bin 26.84 - Beschluß vom 29. November 1984).

Der Antragsteller ist antragsbefugt, da er als Personalrat für die studentischen Beschäftigten an der Technischen Universität auch zuständig ist für die studentischẽn Tutoren, die etwa 950 der etwa 2500 studentischen Beschäftigten ausmachen. Die streitbefangene Fragebogenaktion bezieht sich auch auf Lehrveranstaltungen von Tutoren. 
Die Beschwerde ist begründet. Entgegen dem angefochtenen Beschluß liegen die Voraussetzungen für den Erlaß der begehrten einstweiligen Verfügung nach $\$ 91$ Abs. 2 PersVG Bin in Verbindung mit $\$ 85$ Abs. 2 ArbGG, $\mathbb{\int} 9335,940$ ZPO vor. Im rechtlichen Ausgangspunkt kann dem angefochtenen Beschluß allerdings gefolgt werden: Der Antragsteller begehrt hier die Vorwegnahme der Hauptsache, die im Rahmen des Verfahrens auf Erlaß einer einstweiligen Verfügung nur ausnahmsweise in Betracht kommt, wenn das geltend gemachte Recht im Hauptverfahren nicht mehr durchsetzbar wäre und ein Obsiegen des Antragstellers im Hauptverfahren bei summarischer Prüfung nahezu sicher ist. Diese Voraussetzungen liegen jedoch vor.

Dem Antragsteller steht nach $\$ 85$ Abs. 1 Nr. 13 PersVG Bin ein Mitbestimmungsrecht hinsichtlich der streitbefangenen Maßnahme zu. Danach bestimmt die Personalvertretung mit über die Einführung und Anwendung technischer Einrichtungen, die dazu bestimmt sind, das Verhalten oder die Leistung der Dienstkräfte zu überwachen. Eine technische Einrichtung ist zur Überwachung bestimmt, wenn sie im Rahmen eines auf die Beschäftigten bezogenen Überwachungsvorganges eingesetzt wird, um Fakten oder Daten, denen Bedeutung für die zu überwachende Leistung oder das zu überwachende Verhalten der Beschäftigten zukommt, aufzuzeichnen, zu übermitteln, zu verarbeiten oder auszuwerten (BVerwG 6 P 35.85, Beschluß vom 31. August $1988=$ BVerwGE 80, 143 [146] Buchholz $250 \$ 75$ BPersVG Nr. 63). Im vorliegenden Falle werden die Daten zwar „manuell” durch Ausfüllen der Fragebögen erhoben. Sie werden jedoch dann in die elektrische Datenverarbeitungsanlage eingespeist, um dort gespeichert und verarbeitet zu werden. Auch diese mittelbare Datenerfassung durch die Auswertung „manuell” erhobener Daten durch eine technische Einrichtung ist mitbestimmungspflichtig (BVerwG 6 P 32.84, Beschluß vom 16. Dezember 1987 = Buchholz $250 \$ 75$ BPersVG Nr. 53; BAG AP $\$ 87$ BetrVG 1972 Nr. 9; Grabendorff/Windscheid/Ilbertz/Widmaier, Bundespersonalvertretungsgesetz, 6. Aufl. 1986, $\$ 75$ Rdnr. 206). Zutreffend hebt die Beschwerde hervor, daß zu dem Anwenden der technischen Einrichtung hier sowohl das Speichern als auch das Verarbeiten im Sinne einer Auswertung der erhobenen Daten gehört.

Die Anwendung der technischen Einrichtung ist auch dazu bestimmt, die Leistung der Dienstkräfte zu überwachen. Die Leistungsbezogenheit der einzelnen Fragen ist, wie die eingangs angeführten Beispiele zeigen, offensichtlich. Das unmittelbare Ziel der Fragebogenaktion einschließlich der EDV-gestützten Auswertung der Daten ist es, eine Leistungsbewertung der einzelnen Lehrveranstaltungen aus studentischer Sicht zu erhalten. Ohne Erfolg hält der Beteiligte dies für personalvertretungsrechtlich irrelevant, weil die Studenten nicht Dienstvorgesetzte der Tutoren seien. Ob es - wie der Beteiligte vorträgt - nach dem Arbeits- und nach dem Beamtenrecht geboten ist, Leistungsbewertungen ausschließlich durch den jeweiligen Vorgesetzten vornehmen zu lassen, ist in diesem personalvertretungsrechtlichen Zusammenhang ohne Bedeutung. Entscheidend ist vielmehr, daß hier eine Leistungsbewertung durch die Studenten vorgenommen werden soll, denen der Beteiligte seinerseits Bedeutung für die Lehre zumißt. Auch wenn die Ergebnisse in individualisierter Form nach Darstellung des Beteiligten weder der Personalabteilung noch den Fachbereichen zugänglich gemacht werden, sondern nur den betroffenen Tutoren, ändert dies nichts daran, daß es sich um eine Leistungsüberwachung im Sinne des Personalvertretungsrechts handelt. Es ist erklärtes Ziel des Beteiligten, durch den Rücklauf der gewonnenen Ergebnisse die Tutoren zu einer Überprüfung ihres Lehrverhaltens anzuregen, um gegebenenfalls Korrekturen vorzunehmen und um dadurch insgesamt zu einer besseren Lehre an der Universität beizutragen.

Im übrigen wird nach ganz überwiegender Meinung die Auffassung vertreten, es genüge für das Vorliegen des Mitbestimmungsrechtes, daß die technische Einrichtung abstrakt in der Lage ist, die Überwachung der Dienstkräfte zu ermöglichen, sogenannte objektiv-finale Theorie, unabhängig davon, ob der Überwachungseffekt nach dem Willen des Dienststellenleiters ausgenutzt werden soll oder nicht (vgl. BVerwG 6 P 32.84 vom 16. Dezember 1987, Buchholz $250 \$ 75$ BPersVG Nr. 53; Germelmann, Personalvertretungsgesetz Berlin, $\$ 85$ Rdnr. 173 c; Ilbertz, Personalvertretungsgesetz Berlin, $\$ 85$ Rdnr. 84; Altvater/Bacher/ Sabottig/Schneider/Thiel, Bundespersonalvertretungsgesetz, $\$ 75$ Rdnr. 81; Lorenzen/ Haas/Schmitt, Bundespersonalvertretungsgesetz, $\$ 75$ Rdnr. 195 c; Grabendorff/Windscheid/Ilbertz/Widmaier, a. a. O., $\$ 75$ Rdnr. 205; a. A. Fischer/Goeres, Personalvertretungsrecht des Bundes und der Länder $\$ 75$ Rdnr. 114 a).

Im vorliegenden Falle besteht auch der vom Schutzzweck des Mitbestimmungsrechts nach $\$ 85$ Abs. 1 Nr. 13 PersVG Bin geforderte Bezug zwischen den erhobenen Daten und den. betroffenen einzelnen Dienstkräften. Da die Fragebögen mit der Lehrveranstaltungsnum-

Leistungsbewertung der Lebrveranstaltungen aus studentischer Sicht

Mitbestimmungsrecht.nach objektiv-finaler Theorie

Schutzzweck des

Mitbestimmungsrechts

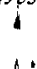




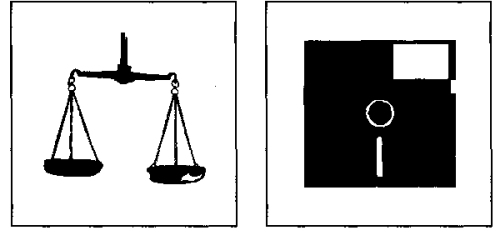

Besonderes Eilbedïrfnis

mer beginnen, können die erhobenen Daten den Lehrveranstaltungen und den dort tätigen Dozenten zugeordnet werden. Auch soweit eine Lehrveranstaltung von mehreren Tutoren betreut werden sollte, handelte es sich wegen der Kleinheit derartiger Gruppen um eine mitbestimmungspflichtige Maßnahme, da sich der Überwachungsdruck auf die Gruppe und deren einzelne Mitglieder auswirken würde (BVerwGE 80, 143; Germelmann, a. a. O., Rdnr. 174 b; Lorenzen/Haas/Schmitt, a. a. O., $\$ 75$ Rdnr. 195 i; Grabendorff/Windscheid/Ilbertz/Widmaier, a. a. O., $\$ 75 \mathrm{Rdnr}$. 206).

Entgegen dem angefochtenen Beschluß liegt im vorliegenden Falle auch ein besonderes Eilbedürfnis vor, das ausnahmsweise die Vorwegnahme des Hauptverfahrens durch die einstweilige Verfügung rechtfertigt. Nach der Darstellung des Beteiligten soll die Fragebogenaktion einschließlich ihrer Auswertung im Herbst 1991 abgeschlossen sein. Bis dahin ist eine Entscheidung im Hauptverfahren nicht zu erwarten. Zutreffend wird im angefochtenen Beschluß zwar darauf hingewiesen, daß die gespeicherten und verarbeiteten Daten bei einem entsprechenden Ausgang eines nachgeholten Mitbestimmungsverfahren gelöscht werden könnten. Zu diesem Zeitpunkt lägen aber bereits die Auswertungen in ausgedruckter Form vor. Der bis dahin eingetretene Überwachungsdruck ließe sich nicht mehr rückgängig machen'.

Gegen diese Entscheidung findet die Rechtsbeschwerde nicht statt, $\mathbb{\$} 92$ Abs. 1 Satz 3, $\mathbb{8} 85$ Abs. 2 ArbGG. 